\title{
Influence of chemical composition on the isothermal cocoa butter crystallization
}

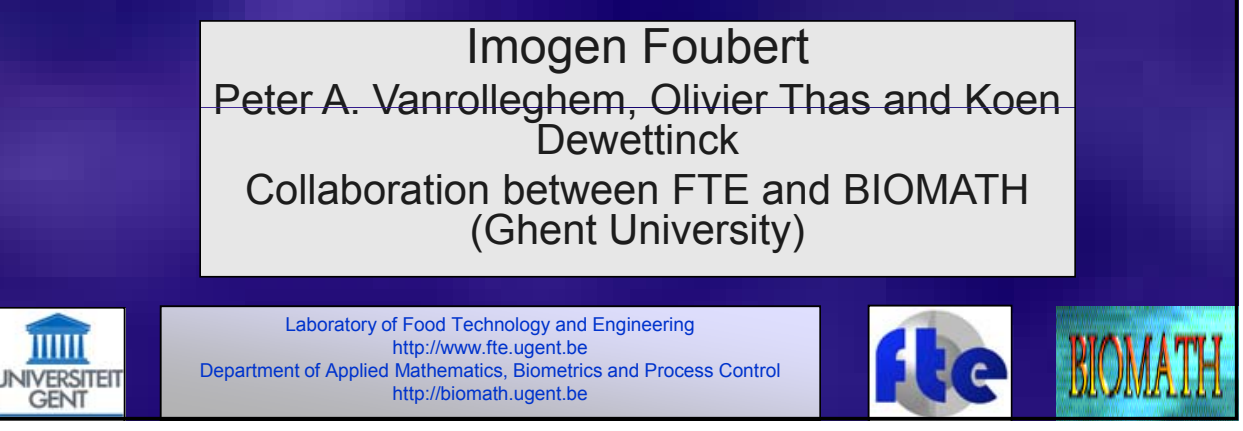

\section{Overview}

- Introduction and aim

- Materials and methods

- What happens during isothermal crystallization, cf. isosbestic point

- Influence of chemical composition on different crystallization parameters

- Take home message

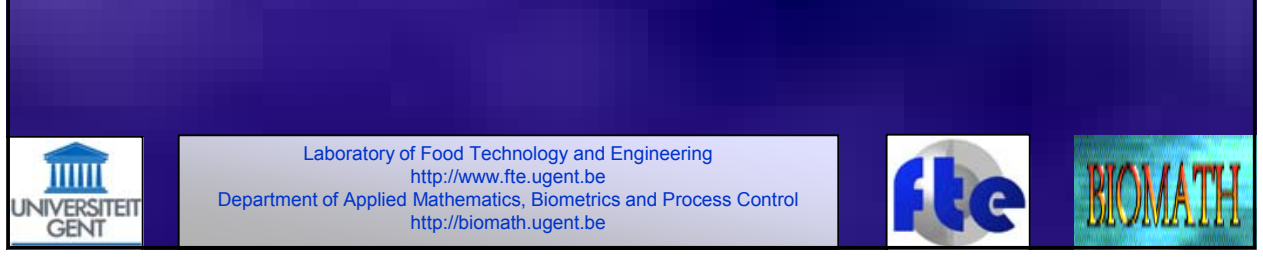




\section{Introduction}

- CHEMICAL COMPOSITION of cocoa butter

- growing conditions, plant age, cacao variety

- production process, refining

- Influence on physical properties, e.g. crystallization kinetics

- => important e.g. for production of chocolate

Laboratory of Food Technology and Engineering

\section{Aim}

- Chemical characterization of twenty cocoa butters

- Quantitative investigation of influence on isothermal, static cocoa butter crystallization described by model of Foubert et al. (2002)

- All different chemical composition variables in one study

- Using biological variability, not by adding chemical substances 


\section{Model of Foubert et al. (2002)}
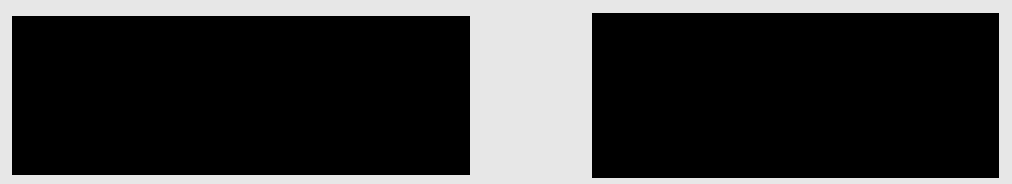

$f(t)$ : amount of crystallization [\%]

$h$ : fraction remaining crystallizable fat $(0<h<1)[-]$

$a_{F}$ : value of $f$ as $t$ approaches infinity [\%]

$\mathrm{K}$ : rate constant $\left[\mathrm{h}^{-1}\right]$

$\mathrm{n}$ : order of the reverse reaction [-]

\section{Model of Foubert et al. (2002)}

- $f(0)$ related to induction time

- physical interpretation of 'induction time' more straightforward + easier to extract from curve $\longrightarrow$ represent as function of induction time

- $t$ ind $d_{x}$ : time needed to reach e.g. $1 \%$ crystallization $(x=0.01)$

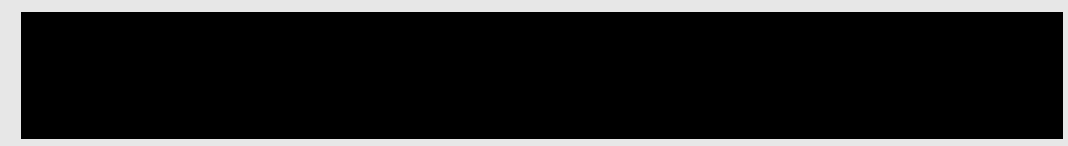




\section{Materials and methods}

- 20 different cocoa butters

- Isothermal crystallization at $20^{\circ} \mathrm{C}$ followed by DSC

- Chemical analyses: fatty acid profile, triglyceride profile, free fatty acids, phosphorus ( phospholipids), iron, traces of soap, unsaponifiable matter, peroxide value, diglycerides

- Principal component analysis: reduce dimensionality of fatty acid and triglyceride profile data

- Multiple linear regression to investigate influence of chemical composition on crystallization properties

\begin{tabular}{|c|c|}
\hline $\begin{array}{l}\text { IIIIIII } \\
\text { IVERSITEIT } \\
\text { GENT }\end{array}$ & $\begin{array}{l}\text { Laboratory of Food Technology and Engineering } \\
\text { http://www.fte.ugent.be } \\
\begin{array}{l}\text { Department of Applied Mathematics, Biometrics and Process Control } \\
\text { http://biomath.ugent.be }\end{array}\end{array}$ \\
\hline
\end{tabular}

\section{Isothermal crystallization at $20^{\circ} \mathrm{C}$}

- Two-step process

- From research with time-resolved X-ray diffraction (synchrotron radiation, ESRF Grenoble)

- Occurence of isosbestic point in SAXS 


\section{Isosbestic point in SAXS}

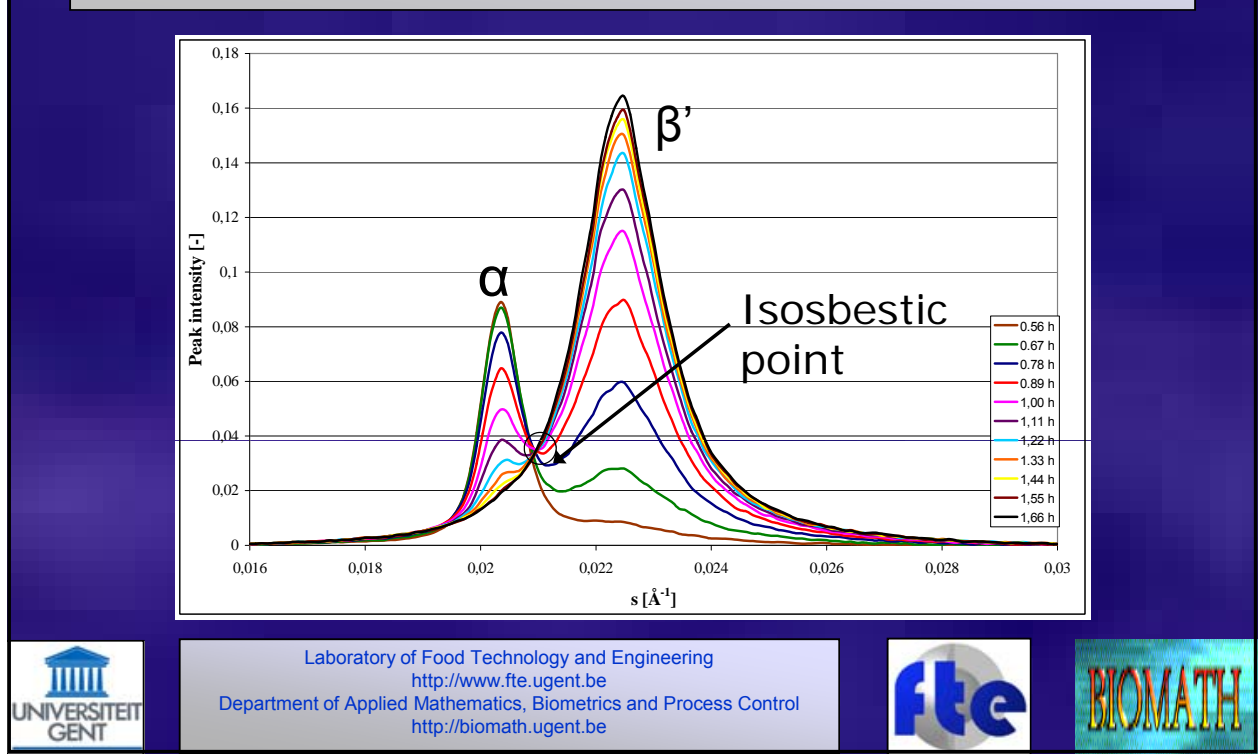

\section{Isothermal crystallization at $20^{\circ} \mathrm{C}$}

- Occurence of isosbestic point in SAXS

- The $\alpha$ structure is transformed into $\beta^{\prime}$ without change of total volume

- End of $\beta^{\prime}$ crystallization coincides with full disappearance of $\alpha$ crystals

- First step: crystallization in a polymorph (kinetically favoured)

- Second step: a mediated $\beta^{\prime}$ crystallization

- No direct $\beta^{\prime}$ crystallization

- Increase in crystallinity during conversion: transformation of liquid-like layer in $\alpha$ 


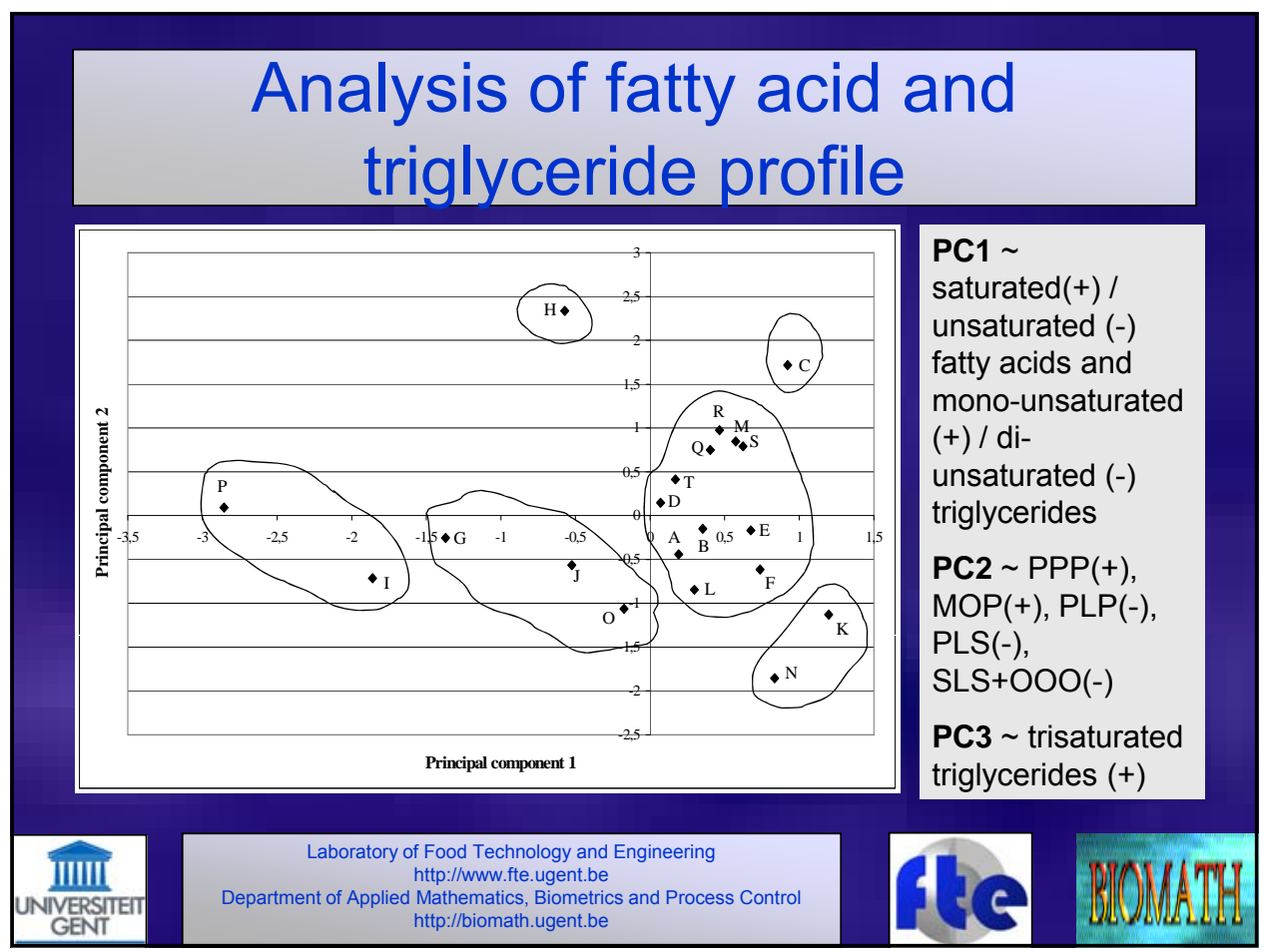

\section{Influence on $\mathrm{a}_{\mathrm{F}}$}

- Amount of crystallization in second step

$\sim$ equilibrium amount of solid fat

- Regression equation: $a_{F}=6.2^{*} P C 1-8.2^{*} F F A-6.8^{*} D G+91$

- + PC1 (Sat, SatUSat versus U, SatUU)

matches results found in literature <= lower percentage

of triglycerides that are able to crystallize

- - free fatty acids

- - diacylglycerols

matches results found in literature on palm oil 


\section{Influence on $t_{-}$ind $d_{x}$}

- Induction time of second step

induction time for polymorphic transition

BUT also growth rate (reverse influence as on $\mathrm{K}$ )

- Regression equation:

t_ind in $_{x}=-0.2^{*} \mathrm{PC} 1-0.06^{*} \mathrm{PC} 3+0.006^{*} \mathrm{P}+0.17^{*} \mathrm{FFA}+0.17^{*} \mathrm{DG}-0.02$

Laboratory of Food Technology and Engineering
http://www.fte.ugent.be
IIIIIII
$\begin{gathered}\text { Department of Applied Mathematics, Biometrics and Process Control } \\ \text { http://biomath.ugent.be }\end{gathered}$

\section{Influence on $t$ _ind ${ }_{x}$}

- - PC1 (Sat, SatUSat versus U, SatUU)

- + phosphorus

- + free fatty acids

- - PC3 (SatSatSat)

- + diacylglycerols

- P, FFA + DG are known to retard polymorphic transition 


\section{Influence on $\mathrm{K}$}

- Rate constant

- Regression equation:

$\mathrm{K}=-1.3^{*} \mathrm{FFA}-2.2^{*} \mathrm{DG}-0.02^{*} \mathrm{PO}-3.8^{*} \mathrm{UM}-0.007^{*} \mathrm{~S}-0.47^{*} \mathrm{PC} 2+0.28^{*} \mathrm{PC} 1+11$

- diacylglycerols

creation irregularities in packing

- - soap

influence known in chocolate industry

IIIIII Laboratory of Food Technology and Engineering

\section{Influence on $\mathrm{K}$}

- - free fatty acids

- - PC2 (PPP, MOP, P versus PLP, PLS, SLS + OOO)

- + PC1 (Sat, SatUSat versus U, SatUU) only crystallization parameter for which not most important influence $<=$ interference of extra oleate chain with molecular packing of mono-unsaturated triglycerides 


\section{Influence on $\mathrm{n}$}

- Order of reverse reaction (local remelting, redissolving of crystals or combination)

$\sim$ how long reverse reaction effects crystallization process: higher $\mathrm{n}$, shorter influence

- Regression equation:

$n=0.9^{*} P C 1+7.3^{*} U M-0.4^{*} \mathrm{Fe}-0.39^{*} \mathrm{DG}+0.17^{*} \mathrm{PO}+0.02^{*} \mathrm{P}+1.9$

Laboratory of Food Technology and Engineering

\section{Influence on $\mathrm{n}$}

- + PC1 (Sat, SatUSat versus U, SatUU)

higher value $=>$ higher melting point $=>$ less effect of remelting $=>$ higher $\mathrm{n}$

- - iron

- + unsaponifiable matter

- + peroxide value

- + phosphorus

- - diacylglycerols

promote nucleation $=>$ larger number of smaller crystals $=>$ redissolving promoted $=>$ lower $n$

Laboratory of Food Technology and Engineering
http://www.fte.ugent.be
$\begin{gathered}\text { IIIIII } \\
\text { GERSIT }\end{gathered}$ \begin{tabular}{c}
$\begin{array}{c}\text { Department of Applied Mathematics, Biometrics and Process Control } \\
\text { http://biomath.ugent.be }\end{array}$ \\
\hline
\end{tabular}




\section{Take home message}

- Most important chemical characteristic with influence in crystallization

- ratio saturated versus unsaturated and monounsaturated versus di-unsaturated (except K)

- most important minor components: free fatty acids and diacylglycerols: negative influence on equilibrium amount of solid fat, growth rate and polymorphic transition

- Other: phospholipids + traces of soap

\section{Acknowledgements}

- DUBBLE

- Bart Goderis (KULeuven)

- FWO-Vlaanderen

- MSc students

- De Smet Engineering ( $\mathrm{P}+\mathrm{Fe}$ analyses)

- Barry Callebaut, ADM (providing samples) 